\title{
Research Paper: Evaluating the Word/Non-Word Repetition in Adults With Stuttering Based on Stuttering Severity and Length of Words/Non-Words
}

\author{
Farhad Choopanian $^{1}$ Q, Morteza Farazi ${ }^{1^{*}}$ Q, Zahrasadat Qoreishi ${ }^{1}$, Atefeh Rahmati ${ }^{1}$, Gholamreza Ghaedamini ${ }^{1}$, Zahra Ilkhani $^{2}$
}

1. Department of Speech Therapy, University of Social Welfare and Rehabilitation Sciences, Tehran, Iran.

2. Department of Speech Therapy, Isfahan University of Medical Sciences. Isfahan, Iran.

$\begin{aligned} & \text { Use your device toscan } \\ & \text { and read the artice online }\end{aligned}$
etition in Adults With Stuttering Based on Stuttering Severity and Length of Words/Non-Words. Journal of Modern Rehabilita-
tion. 2019; 13(4):199-206. http://dx.doi.org/10.32598/JMR.13.4.199
d.t. http://dx.doi.org/10.32598/JMR.13.4.199

Article info:

Received: 16 Jun 2019

Accepted: 27 Aug 2019

Available Online: 01 Oct 2019

\section{Keywords:}

Stuttering, Phonological processing, Stuttering severity, Non word repetition, Word repetition

\section{A B STRACT}

Introduction: Stuttering is a speech disorder examined in many studies on motor speech deficiency in people with stuttering. In the area of language, some studies have also shown that people with stuttering are different from people without stuttering in terms of phonological, lexical, and syntactic processing .In this area, studies on the evaluation of phonological processing in Iranian people with stuttering are a few. One of the tasks for evaluating phonological processing is anon-word repetition task. This study aimed to examine the ability of adults who stutter to repeat words/non-words compared with those with fluent speech.

Materials and Methods: This research is across-sectional descriptive-correlational study. The participants included 20 adults with stuttering (18-30 years old) selected by the convenience sampling method from those referred to speech therapy centers and hospitals in Tehran Province. Besides, 30 age-matched peers participated in this study as the control. The phonological processing of participants was examined in terms of reaction time and word/nonword repetition accuracy. To detect stuttering, we asked the subjects to read a text in Persian, and its severity was detected by stuttering severity instrument-3. For measuring reaction time, DMDX software was used, and data were analyzed in SPSS V. 21

Results: Mann-Whitney test results showed a significant difference between adults with and without stuttering in terms of repetition accuracy $(\mathrm{P}<0.05)$, but no significant difference was found between them in terms of reaction time $(\mathrm{P}>0.05)$.

Conclusion: Phonological processing in adults who stutter is different compared with those with speech fluency, but this difference is non-significant. Results indicated slow phonological processing in an adult with stuttering. This can raise the awareness of therapists during the evaluation and treatment of stutterers in terms of phonological processing and phonological working memory.

* Corresponding Author:

MortezaFarazi, PhD.

Address: Department of Speech Therapy, University of Social Welfare and Rehabilitation Sciences, Tehran, Iran.

Tel: +98 (912) 1405628

E-mail: mfarazi80@gmail.com 


\section{Introduction}

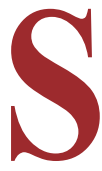

tuttering is a speech disorder that disturbs the timing and coordination of vocal tract, respiratory system, and larynx $[1,2]$. Although the cause of stuttering is unknown, some models have been presented for stuttering, one of which is covert repair hypothesis [3]

According to this theory, people with stuttering have a serious problem in the linguistic system or more precisely in the phonological system, and they are more likely to have a phonological disorder. Deficiency in basic phonological skills is one of the reasons for distinguishing people with stuttering from fluent speakers. A slow phonological encoding process is the cause of more errors in phonetic programming.

Another model of stuttering is called "EXPLAN" based on this model, if speech planning (PLAN) is difficult, it may operate under time pressure, and its Execution (EX) starts when at least a part of the plan is ready for production. In such circumstances, speech production can be delayed until the rest of the plan arrives (stalling), which causes the repetition of the whole word or phrase, interjection, or pause during the speech. Words with simple phonology have a simple plan and high execution speed. During the execution of such simple segments, the time for planning a subsequent word could not be enough if it has complex phonology [4].

According to conducted studies, people with stuttering are different from people without it in terms of phonological, lexical, and syntactic processing [5-7]. Evidence suggests that the speech and language processing system in people with stuttering is slower and less accurate than people without this disorder $[8,9]$. One of the roles of speech and language processing systems is the temporary storage of verbal information (phonological working memory) [10], which is expected to be weaker in people with stuttering than in people without stuttering [11]. Previous studies have suggested that the dysfunction of Phonological Working Memory (PWM) may lead to problems with fluent speech maintenance in people with stuttering [12].

One of the tasks used in assessing the underlying processing system is the Non-Word Repetition (NWR) task, which targets the speech motor system. NWR involves the following stages: auditory processing, phonological analysis, phonological storage and retrieval, speech motor planning, and execution [13].
It is widely used to specifically examine PWM and distinguish people with stuttering from people without it. Hence, PWM plays an essential role in the NR process [14]. Some studies have investigated stuttering by using NWR, motor processing, and linguistic-cognitive processing in adults who stutter $[14,15]$.

Sasisekaran showed PWM deficits in adults with stuttering. Participants were 9 adults with stuttering and 9 normal controls with a mean age of 31 years. The task was the repetition of non-words with different lengths. Those with stuttering showed a lower percentage of accurate repetitions compared with the control group. They also had significantly longer speech durations [13]

In another study on exploring the PWM of adults who stutter through the use of NWR, participants repeated a set of 12 non-words across 4 syllable lengths. Adults with stuttering were significantly less accurate than adults without it in their initial attempts to produce the longest non-words. They had higher attempts to accurately produce non-words than adults who do not stutter [14].

This finding confirms the PWM deficits in people with stuttering. Ludlow et al. studied speech production learning in adults with stuttering on NWR. Participants were 5 adults with stuttering and 5 fluent speakers who repeated two sets of words for several times. Speech fluency of both groups showed better accuracy over time and by the repetition of non-words, although fluent speakers reported more accuracy. This difference between groups was suggested to be the result of deficient phonological encoding in adults with stuttering [15].

The slow speech motor system at the initiation of words and non-words repetition can be examined using the verbal reaction time. People with stuttering have longer reaction times at initiating various speech movements during word or non-word repetition compared with people without stuttering [16].

This difference can be due to the length and complexity of words and non-word sbecause with increasing the complexity of non-words, more areas are involved in the planning and execution of speech movements [17]. Smith et al. investigated the effect of phonological complexity and length of non-words on the production and coordination of adults who stutter. In this regard, the performance of 17 adults who stutter and 17 matched control participants on NWR was assessed. Their results showed that adults who stutter had much less coordination consistency over non-word repetition productions. 
This difference between groups increased with increasing length and complexity of the non-words [18].

Stuttering severity is another factor affecting the verbal reaction time in people with stuttering. In a study to assess the stuttering severity on laryngeal reaction time, Watson and Alfonso showed that in mild stutters, the reaction time did not differ from those with fluent speakers, while severe stutters' reaction time was higher [19].

According to the evidence, the function of verbal components of people with stuttering at the initiation of phonation is associated with delayed and slow reaction times, more variation in movements, and a lack of timing of sequential movements [20]. Moreover, they may have PWM disorder, although it has not yet been conclusively proven. Since so far, little research has been done on PWM impairment in stutterers with varying degrees of dis fluency, the purpose of this study is to evaluate the difference in word/non-word repetition between adults with and without stuttering, and compare stuttering adults based on different stuttering severities and syllable levels.

\section{Methods}

\section{Study participants}

This research is a descriptive/correlational study with a cross-sectional design conducted on 50 adults selected by convenience sampling method. They were divided into stuttering $(n=20)$ and control $(n=30)$ groups. The stuttering group included both male and female adults with developmental stuttering who referred to speech therapy centers and hospitals in Tehran and clinics affiliated to Tehran University of Medical Sciences.

The inclusion criteria included being adults, lacking speech and language disorders such as cluttering, dysarthria, genetic syndromes, etc. (research-based questionnaire), having no physical, neurological and psychiatric disorders according to their medical records and through interview, being right-handed (informal assessment by the examiner), having normal mental abilities, hearing and vision (based on Ling test), and being monolingual Persian-speaking (research-based questionnaire). The exclusion criterion was the unwillingness of the participant to continue the study.

After explaining the purpose of the study to the participants, written informed consent was obtained from them. A speech-language pathologist detected stuttering via an interview with the subjects after reading a text in Persian pro- vided by Farazi et al. [7], and its severity was determined by Stuttering Severity Instrument-3 (SSI-3) tool [21].

We used a set of words and non-words provided by Masumi et al. (2015) for performing the repetition task. They designed in DMDX software [22]. Each word and non-word was presented to the participants in the order of syllable complexity to repeat them. The test was performed in an acoustic room. Auditory stimuli were presented through headphones, and visual stimuli were displayed on a laptop screen; the accuracy of the response and the time it took for the subject to repeat them was measured by DMDX application.

After testing, the recorded data were imported in SPSS V. 21 for analysis. For describing variables, descriptive statistics, including mean and standard deviation, were used, and for testing research hypotheses, non-parametric tests, including Mann-Whitney, Kruskal-Wallis, and Chi-squared tests, were employed. Moreover, since the sample size in each group was less than 50, the Shapiro-Wilk test was performed for testing the normality of data distribution.

\section{Results}

In this study, 20 adults with stuttering (mean age of 25.25 years) and 30 adults without stuttering (mean age of 26.3 years) were studied. Table 1 presents the characteristics of the study participants. Regarding scores reported from SSI-3, participants were divided into different groups in terms of stuttering severity (Table 2). Table 3 presents the mean and standard deviation of reaction time (ms) for words and non-words stimuli in samples with and without stuttering. Table 4 presents the mean and standard deviation of reaction per the number of syllables.

The accuracy of word/non-word repetition was determined as the number (\%) of the correct and incorrect answers (error) in both groups. The number of errors in the repetition of words and non-words was the number of phonological errors during the repetition. Table 5 presents the error percent age of repetition for each group as well as the Chi-squared test results for examining the significant difference between groups in terms of repetition accuracy. Moreover, reaction time to word/ non-word stimuli in adults with and without stuttering (controls) was evaluated using the Mann-Whitney test (Table 6).

Based on the results, no significant difference was found between groups with respect to word repetition 
accuracy $(\mathrm{P}>0.05)$, while the difference was significant in terms of non-word repetition accuracy $(\mathrm{P}<0.05)$. The error percentage of non-word repetition in stuttering adults was higher (with lower accuracy) compared with the control peers.

According to the results in Table 7, the reaction time variable has been normally distributed. To compare reaction time between stuttering adults with three different levels of severity, we used One-way ANOVA. Since $\mathrm{P}>0.05$, there is no significant difference between them.

\section{Discussion}

The purpose of this study was to evaluate the ability to repeat words and non-words in adults with stuttering compared with their normal peers and examining the effect of syllable length and stuttering severity on the speed and accuracy of words/non-words repetition in adults with stuttering. Unlike some studies, our results showed that in people with stuttering, the reaction time to words/non-words repetition is more prolonged than people without stuttering $[16,23]$. In this study, no significant difference was observed between people who stutter and those who do not in terms of reaction time. Probably, the difference between the two groups in repeating non-words versus the repetition of words was due to differences in the methods used to access, retrieve, and execute words compared with those in the non-words [24].

Moreover, phonological decoding and encoding in non-words were different than words which could affect the severity of speech disfluency in people who stutter, because in non-words, the retrieval of semantic content (word meaning) was deleted in comparison with words, and the output of non-words was focused on phonological encoding [25]. Furthermore, the weakness of nonwords repetition in people with stuttering showed their poor PWM [14], but in previous studies, the number of participants was lower compared with our research, which showed the results with less certainty. In our research, word and non-word stimuli were showed the participant using DMDX software. This procedure could

Table 1. Characteristics of the participants

\begin{tabular}{cccc}
\hline Group & No. & Age $(\mathbf{y})$ & Mean \pm SD \\
\hline Control group & 30 & $18-30$ & $26.3 \pm 3.3$ \\
\hline Stuttering group & 20 & $18-30$ & $25.25 \pm 4.575$ \\
\hline
\end{tabular}

$J M R$

Table 2. Frequency of stuttering based on severity levels

\begin{tabular}{cccc}
\hline Stuttering Severity & No. & Reaction Time for Word-Repetition & Reaction Time for Non-Word Repetition \\
\hline Mild & 8 & 1830 & 2060 \\
Moderate & 7 & 1571 & 1775 \\
Severe & 5 & 1864 & 2068 \\
\hline
\end{tabular}

Table 3. The Mean \pm SD of reaction time on word/non-word repetition (ms)

\begin{tabular}{ccc}
\hline Variable & Group & Mean \pm SD \\
\hline Word repetition & Control group & $1859.77 \pm 359.98$ \\
& Stuttering group & $1751.1 \pm 292.04$ \\
\hline Non-word repetition & Control group & $2068.7 \pm 332.47$ \\
& Stuttering group & $1966.9 \pm 295.49$ \\
\hline
\end{tabular}


Table 4. Mean $\pm S D$ of reaction time on word/non-word repetition (number of syllables)

\begin{tabular}{cccc}
\hline & & \multicolumn{2}{c}{ Mean \pm SD } \\
\cline { 3 - 4 } Variable & & Control & Stuttering \\
\hline Words & 1 syllable & $1398 \pm 436$ & $1310 \pm 187$ \\
& 2 syllables & $1725 \pm 379$ & $1589 \pm 329$ \\
& 3 syllables & $1882 \pm 362$ & $1757 \pm 325$ \\
& 4 syllables & $1995 \pm 367$ & $1988 \pm 355$ \\
& 5 syllables & $2236 \pm 295$ & $2095 \pm 385$ \\
& 1 syllable & $1442 \pm 429$ & $1356 \pm 216$ \\
& 2 syllables & $1895 \pm 359$ & $1810 \pm 304$ \\
& 3 syllables & $2119 \pm 343$ & $2035 \pm 370$ \\
& 4 syllables & $2343 \pm 328$ & $2246 \pm 377$ \\
& 5 syllables & $2520 \pm 353$ & $2461 \pm 315$ \\
\hline
\end{tabular}

Table 5. Comparing the accuracy of word/non-word repetition in study groups

\begin{tabular}{|c|c|c|c|c|c|}
\hline Variable & & Group & \% Error & $\chi^{2}$ & $\mathbf{P}$ \\
\hline \multirow{4}{*}{ Repetition accuracy } & \multirow{2}{*}{ Word } & Control group & 0 & \multirow{2}{*}{0.065} & \multirow{2}{*}{1} \\
\hline & & Stuttering group & 0 & & \\
\hline & \multirow{2}{*}{ Non-word } & Control group & 44.8 & \multirow{2}{*}{6.062} & \multirow{2}{*}{0.014} \\
\hline & & Stuttering group & 80 & & \\
\hline
\end{tabular}

Table 6. Comparing reaction time on word/non-word repetition between study groups

\begin{tabular}{rccccc}
\hline Variable & & Group & Mean Rank & Z & P \\
\hline & \multirow{2}{*}{ Word } & Control group & 22.41 & -0.643 & 0.520 \\
Reaction time & & Stuttering group & 19.87 & & \\
& \multirow{2}{*}{ Non-word } & Control group & 22.22 & & \\
& & Stuttering group & 20.20 & -0.512 & 0.609 \\
\hline
\end{tabular}

Table 7. Testinghomogeneity of reaction time variance based on stuttering severity levels

\begin{tabular}{|c|c|c|c|c|}
\hline & Variable & Stuttering Severity & Statistic & $\mathbf{P}$ \\
\hline \multirow{6}{*}{ Reaction time } & \multirow{3}{*}{ Word repetition } & Mild & & \multirow{3}{*}{0.448} \\
\hline & & Moderate & 0.86 & \\
\hline & & Severe & & \\
\hline & \multirow{3}{*}{ Non-word repetition } & Mild & & \multirow{3}{*}{0.287} \\
\hline & & Moderate & 1.387 & \\
\hline & & Severe & & \\
\hline
\end{tabular}


reduce the amount of environmental errors and individuals' interference.

Regarding repetition accuracy, no difference was reported between study groups in terms of word repetition, but adults with stuttering had significantly lower accuracy of nonword repetition than normal peers. This finding is consistent with the findings of Pelczarski [26] and Smith et al. [18].

It seems that people who stutter rely more on semantic information to make up for poor performance in other aspects of phonological coding. Therefore, this difference can be due to poorer phonological coding in stutterers than those with speech fluency [26], but as stated, these differences also depend on the length and complexity of words and non-words [17].

For example, Smith et al. studied the effect of phonological complexity and length of non-words on inter-articulator coordination. They reported that adults who stutter were much less consistent in their coordinative patterns over repeated productions, and with increasing length and complexity of the non-words, these differences increase. In their study, both adults with and with out stuttering showed a higher error rate with the increase of length and complexity of the non-words, and the accuracy of their responses reduced. This can be due to more difficult phonological retrieval in individuals [18].

Byrd et al. studied the PWM of adults who stutter. Their study participants were 10 adults who stutter and 10 matched peers tested by using non-word repetition and compared as vocal versus non-vocal. They found out that the adults who stutter needed fewer accurate initial productions of7-syllable non-words than those who do not stutter [14]. Our results are consistent with the results of Byrd et al. and showed that with the increasing number of syllables, the accuracy of production decreases.

Zangeneh et al. reported that the frequency of non-fluency on words and non-words in adults who stutter was significantly higher, which is consistent with our results [23]. Our results indicated that the error percentage of people with stuttering in repeating non-words was significantly higher compared with their matched peers. In previous studies, the words/non-words repetition has not been compared among adults with different stuttering severity. In this study, this comparison was carried out, and results revealed that the difference between various levels of stuttering severity was not significant in terms of reaction time and repetition accuracy. Based on our results, phonological processing is different in people with stuttering and those with speech flu- ency, but this difference was not significant. Further studies are needed in this area.

\section{Conclusion}

The ability of adults who stutter in performing word/nonword repetition was weaker than people with fluent speech. Reaction time on word/non-word repetition in adults with and without stuttering was not significantly different. Word repetition accuracy of adults who stutter had no significant difference with that of those who do not stutter, while their non-word repetition accuracy was significantly different. Regarding the effect of the number of syllables, it was found out that the function of adults with and without stuttering in repeating longer words/non-words was poorer compared with their function in the repetition of shorter words/nonwords. The difference between adults with different stuttering severity also was not significant with respect to reaction time and repetition accuracy.

\section{Ethical Considerations}

\section{Compliance with ethical guidelines}

The Ethics Committee of the University of Social Welfare and Rehabilitation Sciences approved the study.

Funding

This article is extracted from an MSc. thesis of the first author, Department of Speech Therapy, University of Social Welfare and Rehabilitation Sciences, approved at the University of Social Welfare and Rehabilitation Sciences, Tehran.

\section{Authors contributions}

Conceptualization: Farhad Choopanian, Morteza Farazi, Zahrasadat Qoreishi; Writing the draft: Farhad Choopanian; Investigation, resources, data curation: Farhad Choopanian, Atefeh Rahmati, Zahra Ilkhani; Software, formal analysis: Gholamreza Ghaedamini; Writing the review and editing: Morteza Farazi, Zahrasadat Qoreishi, Atefeh Rahmati.

\section{Conflict of interest}

The authors declared no conflict of interest.

\section{Acknowledgements}

The authors are grateful to the CEOs of the health centers for their kind help with conducting this study. 


\section{References}

[1] Kent RD. Research on speech motor control and its disorders: A review and prospective. Journal of Communication Disorders. 2000; 33(5):391-428. [DOI:10.1016/S0021-9924(00)00023-X]

[2] van Lieshout PHHM, Hulstijn W, Peters HFM. Searching for the weak link in the speech production chain of people who stutter: A motor skill approach. In: Maassen B, editor. Speech Motor Control in Normal and Disordered Speech. Oxford: Oxford University Press; 2004. https://books.google.com/ books?lr=\&id=KbVrAAAAMAAJ\&dq

[3] Brocklehurst PH. A review of evidence for the covert repair hypothesis of stuttering. Contemporary Issues in Communication Science and Disorders. 2008; 35:25-43. [DOI:10.1044/ cicsd_35_S_25]

[4] Fava E, editor. Clinical linguistics: Theory and applications in speech pathology and therapy. Amsterdam: John Benjamins Publishing Company; 2002. [DOI:10.1075/cilt.227]

[5] Anderson JD, Byrd CT. Phonotactic probability effects in children who stutter. Journal of Speech, Language, and Hearing Research. 2008; 51(4):851-66. [DOI:10.1044/1092-4388(2008/062)]

[6] Ntourou K, Conture EG, Lipsey MW. Language abilities of children who stutter: A meta-analytical review. American Journal of Speech-Language Pathology. 2011; 20(3):163-79. [DOI:10.1044/1058-0360(2011/09-0102)]

[7] Farazi M, Gholami Tehrani L, Khodabakhshi Koolaee A, Shemshadi $\mathrm{H}$, Rahgozar $\mathrm{M}$. [The effect of a combination of cognitive therapy and speech in stutterers (Persian)]. Thought and Behavior in Clinical Psychology. 2014; 8(30):37-46. https:/ / www.magiran.com/paper/1319998

[8] Newman RS, Ratner NB. The role of selected lexical factors on confrontation naming accuracy, speed, and fluency in adults who do and do not stutter. Journal of Speech, Language, and Hearing Research. 2007; 50(1):196-213. [DOI:10.1044/10924388(2007/016)]

[9] Sasisekaran J, De Nil LF, Smyth R, Johnson C. Phonological encoding in the silent speech of persons who stutter. Journal of Fluency Disorders. 2006; 31(1):1-21. [DOI:10.1016/j. jfludis.2005.11.005] [PMID]

[10] Montgomery JW, Magimairaj BM, Finney MC. Working memory and specific language impairment: An update on the relation and perspectives on assessment and treatment. American Journal of Speech-Language Pathology. 2010; 19(1):78-94. [DOI:10.1044/1058-0360(2009/09-0028)]

[11] Anderson JD, Wagovich SA. Relationships among linguistic processing speed, phonological working memory, and attention in children who stutter. Journal of Fluency Disorders. 2010; 35(3):216-34. [DOI:10.1016/j.jfludis.2010.04.003] [PMID] [PMCID]

[12] Bajaj A. Working memory involvement in stuttering: Exploring the evidence and research implications. Journal of Fluency Disorders. 2007; 32(3):218-38. [DOI:10.1016/j.jfludis.2007.03.002] [PMID]

[13] Sasisekaran J. Nonword repetition and nonword reading abilities in adults who do and do not stutter. Journal of Fluency Disorders. 2013; 38(3):275-89. [DOI:10.1016/j.jfludis.2013.06.001] [PMID] [PMCID]
[14] Byrd CT, Vallely M, Anderson JD, Sussman H. Nonword repetition and phoneme elision in adults who do and do not stutter. Journal of Fluency Disorders. 2012;37(3):188-201. [DOI:10.1016/j. jfludis.2012.03.003] [PMID]

[15] Ludlow C, Siren K, Zikria M, Hulstijn W, Peters H, van Lieshout P. Speech production learning in adults with chronic developmental stuttering. In: Hulstijn W, Peters HFM, Van Lieshout PHHM, editors. Speech Production: Motor Control, Brain Research and Fluency Disorders. Amsterdam: Elsevier; 1997. https://books.google.com/books?id=Xa5rAAAAMAAJ\&dq

[16] Logan KJ. The effect of syntactic structure upon speech initiation times of stuttering and nonstuttering speakers. Journal of Fluency Disorders. 2003; 28(1):17-35. [DOI:10.1016/S0094730X(03)00003-2]

[17] Riecker A, Brendel B, Ziegler W, Erb M, Ackermann H. The influence of syllable onset complexity and syllable frequency on speech motor control. Brain and Language. 2008; 107(2):102-13 [DOI:10.1016/j.bandl.2008.01.008] [PMID]

[18] Smith A, Sadagopan N, Walsh B, Weber-Fox C. Increasing phonological complexity reveals heightened instability in interarticulatory coordination in adults who stutter. Journal of Fluency Disorders. 2010; 35(1):1-18. [DOI:10.1016/j.jfludis.2009.12.001] [PMID] [PMCID]

[19] Watson BC, Alfonso PJ. Foreperiod and stuttering severity effects on acoustic laryngeal reaction time. Journal of Fluency Disorders. 1983; 8(3):183-205. [DOI:10.1016/0094-730X(83)90033-5]

[20] Loucks TM, Luc F, Sasisekaran J. Jaw-phonatory coordination in chronic developmental stuttering. Journal of Communication Disorders. 2007; 40(3):257-72. [DOI:10.1016/j.jcomdis.2006.06.016] [PMID]

[21] Bakhtiar M, Seifpanahi S, Ansari H, Ghanadzade M, Packman A. Investigation of the reliability of the SSI-3 for preschool Persian-speaking children who stutter. Journal of Fluency Disorders. 2010; 35(2):87-91. [DOI:10.1016/j.jfludis.2010.02.003] [PMID]

[22] Forster, K. I., Forster, J.C. (2012). DMDX (Version 4.0.6.0) [Computer software]. Retrieved from http://www.u.arizona. edu/ jforster/dmdx.htm

[23] Mozafar Zangeneh A, Pooladi Sh, Mahmoudi Bakhtiari B, Haghani H. [Investigation of nonfluency frequency on words and nonwords in Persian speaking adults who stutter (Persian)] Research in Rehabilitation Sciences. 2012; 8(5):905-12. http:/ /jrrs mui.ac.ir/index.php/jrrs/article/view/529

[24] Dayalu VN, Kalinowski J, Stuart A. Stuttering frequency on meaningful and nonmeaningful words in adults who stutter. Folia Phoniatrica et Logopaedica. 2005; 57(4):193-201. [DOI:10.1159/000085187] [PMID]

[25] Au-Yeung J, Howell P. Non-word reading, lexical retrieval and stuttering: Comments on Packman, Onslow, Coombes and Goodwin (2001). Clinical Linguistics \& Phonetics. 2002; 16(4):28793. [DOI:10.1080/02699200210128981] [PMID]

[26] Pelczarski K. Phonological processing abilities of adults who stutter [PhD dissertation]. Pittsburgh, PA: University of Pittsburgh; 2011. http://d-scholarship.pitt.edu/10735/ 
This Page Intentionally Left Blank 